\title{
Amoeba co-culture of soil specimens recovered 33 different bacteria, including four new species and Streptococcus pneumoniae
}

\author{
Correspondence \\ Bernard La Scola \\ bernard.lascola@univmed.fr
}

Received 5 August 2008

Revised 6 October 2008

Accepted 13 October 2008

\author{
Anna Evstigneeva, ${ }^{1,2}$ Didier Raoult, ${ }^{1}$ Lev Karpachevskiy ${ }^{2}$ \\ and Bernard La Scola ${ }^{1}$
}

\author{
${ }^{1}$ Unité des Rickettsies, CNRS UMR 6020, Faculté de Médecine de Marseille, 13385 Marseille \\ Cedex 05, France \\ ${ }^{2}$ Moscow State University, Faculty of Soil Science, Leninskie Gory 119899, Russia
}

\section{INTRODUCTION}

Free-living amoebae are important predators that control microbial communities. They are ubiquitous, and have been isolated from various natural sources, such as soil, freshwater, salt water, dust and air. These organisms are also frequently isolated from anthropogenic ecosystems, such as tap water, air-conditioning units and cooling towers, where they feed on the microbial biofilm present in the systems. However, several bacteria have developed mechanisms for surviving phagocytosis by free-living amoebae, and are able to exploit the amoebae as hosts (Molmeret et al., 2005).

Some of these bacteria are pathogenic in humans, and amoebae have been suggested as representative environmental reservoirs that act as 'Trojan horses' of the microbial world (Barker \& Brown, 1994). As a result of this role, amoebae have attracted the attention of clinical microbiologists during recent decades. In general, the current knowledge of the roles played by free-living amoebae, in addition to the role described above, can be presented in the following terms: they can aid in

Abbreviation: ARB, amoeba-resistant bacteria. transmission, selection of virulence traits, and adaptation of the microbes to macrophages (Greub \& Raoult, 2004).

Free-living amoebae may be used as a tool for isolation of intracellular micro-organisms from soil microbial communities, where complex ecological interactions exist between bacteria, viruses, plants, nematodes, microarthropods and protozoa (Brown \& Barker, 1999). Until recently, the ability to monitor the microbial community structure was limited by the lack of a suitable cultivation strategy to define the composition of species, and the relative abundance of specific populations, in microbial communities (Crawford, 2005). The method of amoebal coculture has been used to discover many previously unknown micro-organisms (Adekambi et al., 2004; Adeleke et al., 2001; Birtles et al., 2000; Greub \& Raoult, 2003; La Scola et al., 2000, 2003, 2004a, b), as well as to observe a new niche for survival of pathogenic microorganisms in water (Abd et al., 2005; Greub \& Raoult, 2002; La Scola \& Raoult, 2001; Landers et al., 2000). We use the term amoeba-resistant bacteria (ARB) instead of endosymbionts, since many of the bacteria able to resist destruction by free-living amoebae do not represent true endosymbionts, and it is also the case that endosymbionts 
may be endosymbiotic or lytic in a given amoeba, depending on the environmental conditions.

Here, we present data from what we believe is the first study to use amoeba co-culture to test soil and sand for the presence of ARB. With the objective of identifying new potential human pathogens, we focused our research on soil and sand sources in human environments.

\section{METHODS}

Sample collection and preparation. Altogether, 11 samples were studied: eight soil samples and three sand samples, which were all collected in the city of Marseilles, France. Composite $0.5 \mathrm{~kg}$ samples were collected from the top layer $(0-10 \mathrm{~cm})$ of nine urban soils and three sands (Table 1). All samples were homogenized, and then passed through a $2 \mathrm{~mm}$ sieve prior to analysis. Samples were prepared by dilution in sterile distilled water at a ratio of one to five, with the following three-step filtration procedure: first, they were passed through a $40 \mu \mathrm{m}$ Falcon cell strainer, then through paper filters, with a final filtration through a membrane with $0.22 \mu \mathrm{m}$ pore size. Each membrane was shaken in $2 \mathrm{ml}$ Page's modified Neff's amoeba saline (PAS). This suspension was used for inoculation of amoebal microplates.

Amoeba suspension inoculation. Sample extracts were cocultivated with a PAS suspension of Acanthamoeba polyphaga (strain Linc-AP1) at a concentration of $5 \times 10^{5}$ amoebae $\mathrm{ml}^{-1}$ in 12-well Costar microplates (Corning) (La Scola et al., 2000). Each well contained $1 \mathrm{ml}$ amoeba suspension, and was inoculated with $100 \mu \mathrm{l}$ of each prepared specimen. After inoculation, microplates were centrifuged at 2500 r.p.m. $(500 \mathrm{~g})$ for $30 \mathrm{~min}$, and then incubated at $32{ }^{\circ} \mathrm{C}$ in a humidified atmosphere.

For each specimen, three different series (each containing a negative control) of co-culture were performed: (i) without antibiotics; (ii) with $10 \mu \mathrm{l}$ colistin (Aventis Pharma) and $10 \mu \mathrm{l}$ vancomycin (vancomycin chlorhydrate; Merck) at concentrations of 500 units $\mathrm{ml}^{-1}$ and $10 \mu \mathrm{g} \mathrm{ml}^{-1}$, respectively; and (iii) with $10 \mu \mathrm{l}$ erythromycin (Dakota Pharm) at $4 \mu \mathrm{g} \mathrm{ml}^{-1}$, in addition to the concentrations of colistin and vancomycin described above. Samples without antibiotics were identified by using numbers. The letters CV were added to identify samples containing colistin and vancomycin, and the letter $\mathrm{E}$ was added to identify samples containing colistin, vancomycin and erythromycin. The antibiotics were used to help prevent the excessive growth of extracellular micro-organisms, without suppressing the growth of intracellular micro-organisms (La Scola et al., 2000). The addition of erythromycin inhibits the activity of Legionella spp.; when these organisms are present in a sample, their lytic activity does not allow other species of microorganisms to multiply.

Detection of bacterial growth. The amoebal co-cultures were subcultured on day 3 into wells containing fresh amoebae, and then examined daily for the appearance of amoebal lysis, which suggested the presence of an amoeba-resistant micro-organism. Each well was also screened for the presence of intra-amoebal bacteria. On day 8 , the co-culture suspension (100 $\mu \mathrm{l}$ co-culture, and $200 \mu \mathrm{l} \mathrm{PAS})$ was cytocentrifuged, and slides were stained by using Gram, Gimenez and Ziehl-Neelsen stains. At the same time, a $50 \mu \mathrm{l}$ suspension of coculture from each well was subcultured onto agar media. We used three different media: (i) buffered charcoal yeast extract (BCYE) agar (bioMérieux) enriched with Legionellae supplement (provided by the manufacturer) for the specimens containing antibiotics, (ii) BMPA (BCYE with cefamandole, polymyxin B and anisomycin) agar plates (Oxoid) for the specimens from wells that did not contain antibiotics, and (iii) Columbia agar plates with $5 \%$ sheep blood (trypticase soy agar; bioMérieux) for specimens from wells with and without antibiotics. All agar plates were incubated at a temperature of $32{ }^{\circ} \mathrm{C}$, and they were screened for growing bacteria every 2 days for up to 10 days. Incubation of BCYE agar plates was carried out in a $\mathrm{CO}_{2}$ atmosphere of 3.5-9.5\% (GENbag $\mathrm{CO}_{2}$; bioMérieux). When growth appeared, each different colony was subcultured onto BCYE, BMPA or Columbia agar, as appropriate, and incubated under the appropriate conditions in order to obtain visible colonies. Slides for Gimenez and Gram staining (which also served as verification that the colonies contained a single strain only), and bacterial suspensions (in sterile water) for molecular identification, were prepared from each colony. The remainder of each specimen, and all the strains isolated, were preserved at $-80{ }^{\circ} \mathrm{C}$.

Cytopathogenic testing of isolates. The lytic ability of each isolate towards amoebae was then checked to verify that the agar-grown bacterium was responsible for amoebal lysis. One colony of each strain was suspended in $1 \mathrm{ml}$ PAS, then $50 \mu \mathrm{l}$ of this suspension was added to $1 \mathrm{ml}$ amoebal suspension in a 12-well Costar microplate. The behaviour of the amoebae was examined every day using an inverted microscope. On days 2 and 6 , the suspension $(150 \mu \mathrm{l}$ bacteria-amoeba co-culture and $150 \mu \mathrm{l}$ PAS) was cytocentrifuged, and slides were treated with Gimenez stain.

Molecular identification of isolates. Bacterial DNA was extracted from five colonies suspended in $300 \mu \mathrm{l}$ sterile distilled water, using the QIAamp DNA Minikit (Qiagen), according to the manufacturer's recommendations. For all strains, $16 \mathrm{~S}$ rRNA gene fragments were amplified by PCR using oligonucleotide primers $\mathrm{fD} 1$ and $\mathrm{rP} 2$, and sequenced using the internal primers $536 \mathrm{~F}$ and 1050R, under previously described conditions (La Scola et al., 1997). For isolates that were not expected to be isolated from environmental locations, and for which $16 \mathrm{~S}$ rRNA sequencing was not distinctive enough for species identification, a portion of the coding region of the $r p o B$ gene was also sequenced. For Klebsiella variicola, we used CM7-CM31b and CM 81b-CM 32b primer pairs (amplicon size $1000 \mathrm{bp}$ ) (Mollet et al., 1997); for Streptococcus pneumoniae, we used the rpoBpneumoR-rpoBpneumoF primer pair (amplicon size $740 \mathrm{bp}$ ) (Drancourt et al., 2004). Sequences were compared with those available in the GenBank database using the BLAST program (http:// www.ncbi.nlm.nih.gov/BLAST/).

\section{RESULTS}

\section{Diversity of ARB}

More than 200 strains of ARB were isolated from all the sources studied. After excluding repeat isolates of the same strain on the basis of $16 \mathrm{~S}$ rRNA gene sequence, and checking of each strain for the occurrence of amoebal lysis, a total of 33 different species of ARB were isolated from soil and sand samples (Table 1). The species belonged to four phyla, seven classes and 14 orders. Soil and sand samples harboured approximately the same numerical compositions of ARB (approx. 4.3 species per sample versus 5 per sample, respectively).

\section{Amoebal lysis of specimens, and cytopathogenic effect of isolates}

Among 11 strains inoculated into amoebae, one was characterized by complete amoebal lysis after 3 days, seven 
Table 1. List and taxonomic classification of isolated ARB according to $16 \mathrm{~S}$ rRNA gene sequencing

Species reported to be human pathogens are in bold. Lytic ability is indicated as: C, complete lysis after 24 h; C3, complete lysis after 3 days; C5, complete lysis after 5 days; P, partial lysis after 5 days; G, intra-amoebal growth. Samples are as follows: 1, soil Université de la Mediterranée, Faculté de Médecine; 2, soil Hôpital de la Timone; 3, soil Hôpital de la Conception; 4, soil Jardin Guy Azais; 5 and 6, Parc Borély sand and soil, respectively; 7, sand Plage Borrely; 8, sand Plage de la Pointe Rouge; 9, soil Ferme Pédagogique; 10, soil Parc du XXVI Centenaire; 11, soil Gare St Charles. CV, colistin and vancomycin; E, colistin, vancomycin and erythromycin.

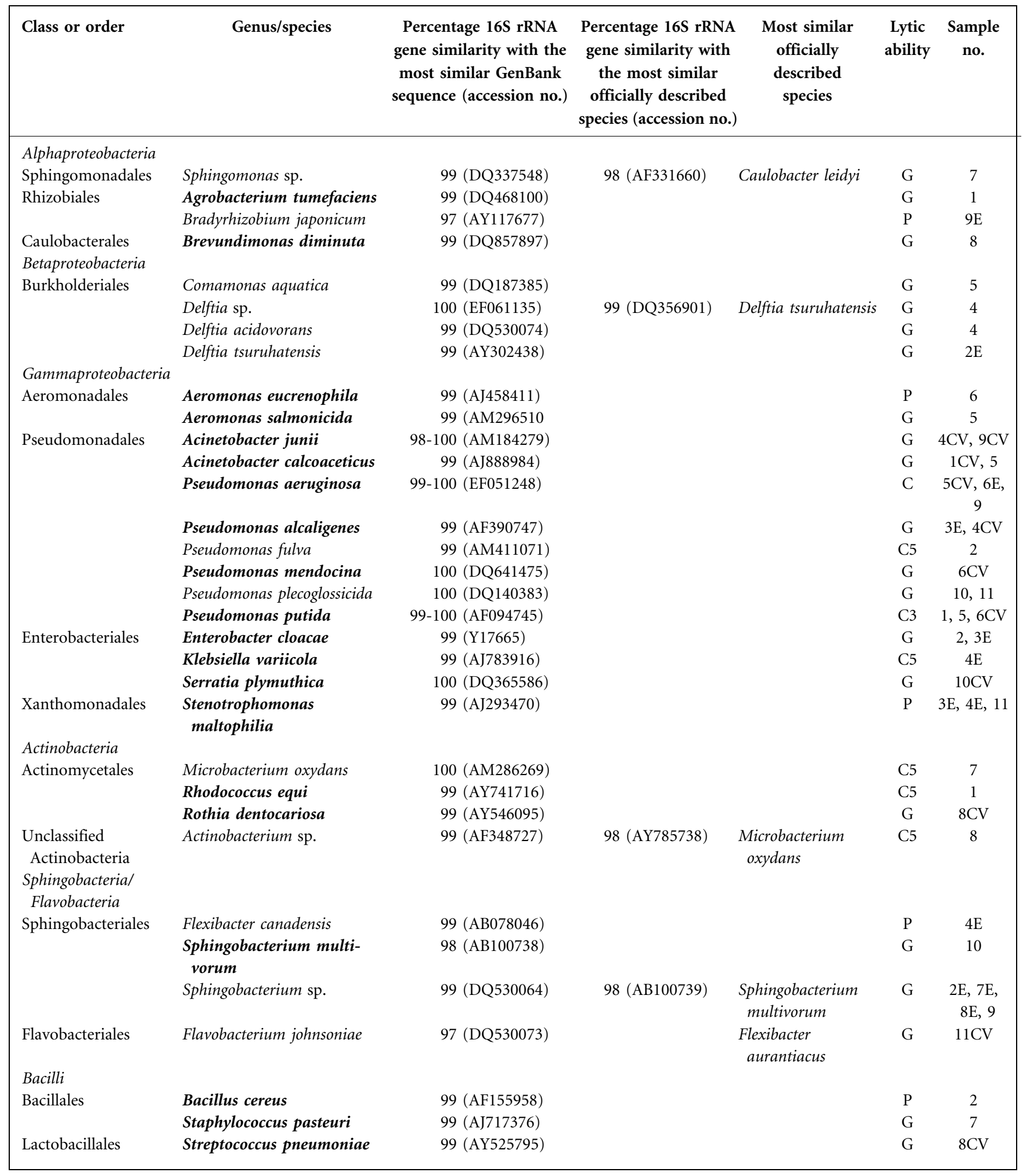


were characterized by complete or partial amoebal lysis after 8 days, and three were characterized by co-existence of amoebae and bacteria. Co-existence was said to have occurred when there was no noticeable temporal change in the number of bacteria or amoeba compared with intraamoebal growth for which we were able to observe increasing quantities of bacteria.

Among 33 isolated strains, $12(\sim 36 \%)$ provoked amoebal lysis: one after $24 \mathrm{~h}$, one after 3 days, and five after 5 days, while five strains produced partial amoebal lysis $(>50 \%$ amoebae were lysed) after 5 days. All the 12 lytic bacterial strains were isolated from the nine soil or sand suspensions for which complete or partial lysis was recorded. The other 21 strains of ARB ( $64 \%)$ demonstrated intra-amoebal growth.

\section{Identification of isolates}

The bacteria were mostly Gram-negative. Three isolates were Gram-positive: Staphylococcus pasteuri, Rothia dentocariosa and Str. pneumoniae. The 16S rRNA gene sequences allowed good identification of 29 isolates that showed $\geqslant 99 \%$ similarity to known species. However, four isolates exhibited $\leqslant 98 \%$ 16S rRNA gene similarity to known species, even after determination of almost the complete $16 \mathrm{~S}$ rRNA gene sequence (Table 2, Fig. 1). The identification of Str. pneumoniae was confirmed by use of $r p o B$ gene partial sequencing (720 bp with a similarity of $99 \%$ ). Definitive identification of Klebsiella variicola and Klebsiella singaporensis (not distinguishable from Klebsiella pneumoniae using 16S rRNA sequencing) was also performed using $r p o B$ partial gene sequencing (Fig. 2). The number of ARB isolates that have been described as human pathogens was approximately equal for soil and sand samples (2.8 species per soil sample versus 2.3 per sand sample). The highest number of pathogens (four species) was isolated from samples 1,4 and 6 , which were collected from near the university, in the square and in the park, respectively.

\section{DISCUSSION}

Until now, the majority of bacteria-protozoa interaction models have focused on cooling tower and hospital water supplies. However, as noticed by Snelling et al. (2006), $16 \mathrm{~S}$ and $18 \mathrm{~S}$ rRNA studies are urgently required to catalogue the population diversity of bacteria and protozoa in different water types, as well as in different types of soils. Soil is believed to be one of the most complex environments for microbial life, as it contains about $10^{9}$ bacterial cells $\mathrm{g}^{-1}$, and an estimated $10^{4}$ distinct species (Crawford, 2005). Culturing the total diversity of species currently seems to be an unachievable goal, given that such large numbers of isolates are not routinely cultured and identified (Janssen, 2006). We believe that ARB, by their capability to survive within phagocytes, are potential human pathogens. This hypothesis is supported by the fact that Legionella have similar cytotoxic defects and intracellular replication in mammalian macrophages and protozoa, suggesting a common adaptive mechanism to the intracellular environment. Moreover, strains of Acanthamoeba spp. have been found to produce Legionella-containing vesicles, which may be agents of transmission of legionellosis. While Legionella has been the most studied ARB to date, we have demonstrated that several previously unidentified ARB are also human pathogens (Greub et al., 2004; La Scola et al., 2001, 2003).

The aim of our project was to identify ARB in the natural environment surrounding humans, and thus detect possible emerging human pathogens or possible new reservoirs of known pathogens. In order to do this we tested soil and sand sources, and, from the results described herein, we can confirm that amoebal co-culture is a pertinent tool to achieve this goal. In this work, the ARB isolates belonged to the phyla Proteobacteria, Actinobacteria, Bacteroidetes and Firmicutes These four phyla, together with five others (Acidobacteria, Verrucomicrobia, Chloroflexi, Planctomycetes and Gemmatimonadetes), have been demonstrated to be dominant in bacterial

Table 2. Proposed names and $16 \mathrm{~S}$ rRNA gene sequence accession numbers of isolates proposed as new species

\begin{tabular}{|c|c|c|c|c|c|c|}
\hline Phylum & Class & Order & Genus and species & $\begin{array}{l}\text { 16S rRNA gene } \\
\text { similarity (\%) }\end{array}$ & Proposed name & $\begin{array}{c}\text { GenBank } \\
\text { accession no. }\end{array}$ \\
\hline \multirow[t]{2}{*}{ Proteobacteria } & Alphaproteobacteria & Rhizobiales & Bradyrhizobium japonicum & 97 & $\begin{array}{l}\text { 'Candidatus } \\
\text { Bradyrhizobium } \\
\text { massiliensis' }\end{array}$ & EF203409 \\
\hline & Gammaproteobacteria & Pseudomonadales & Acinetobacter junii & 98 & $\begin{array}{l}\text { 'Candidatus } \\
\text { Acinetobacter } \\
\text { timonensis' }\end{array}$ & EF203410 \\
\hline \multirow[t]{2}{*}{ Bacteroidetes } & Flavobacteria & & Flavobacterium johnsoniae & 97 & $\begin{array}{l}\text { 'Candidatus } \\
\text { Flavobacterium } \\
\text { massiliensis' }\end{array}$ & EF203412 \\
\hline & Sphingobacteria & & $\begin{array}{l}\text { Sphingobacterium } \\
\text { multivorum }\end{array}$ & 98 & $\begin{array}{l}\text { 'Candidatus } \\
\text { Sphingobacterium } \\
\text { massiliensis' }\end{array}$ & EF203411 \\
\hline
\end{tabular}




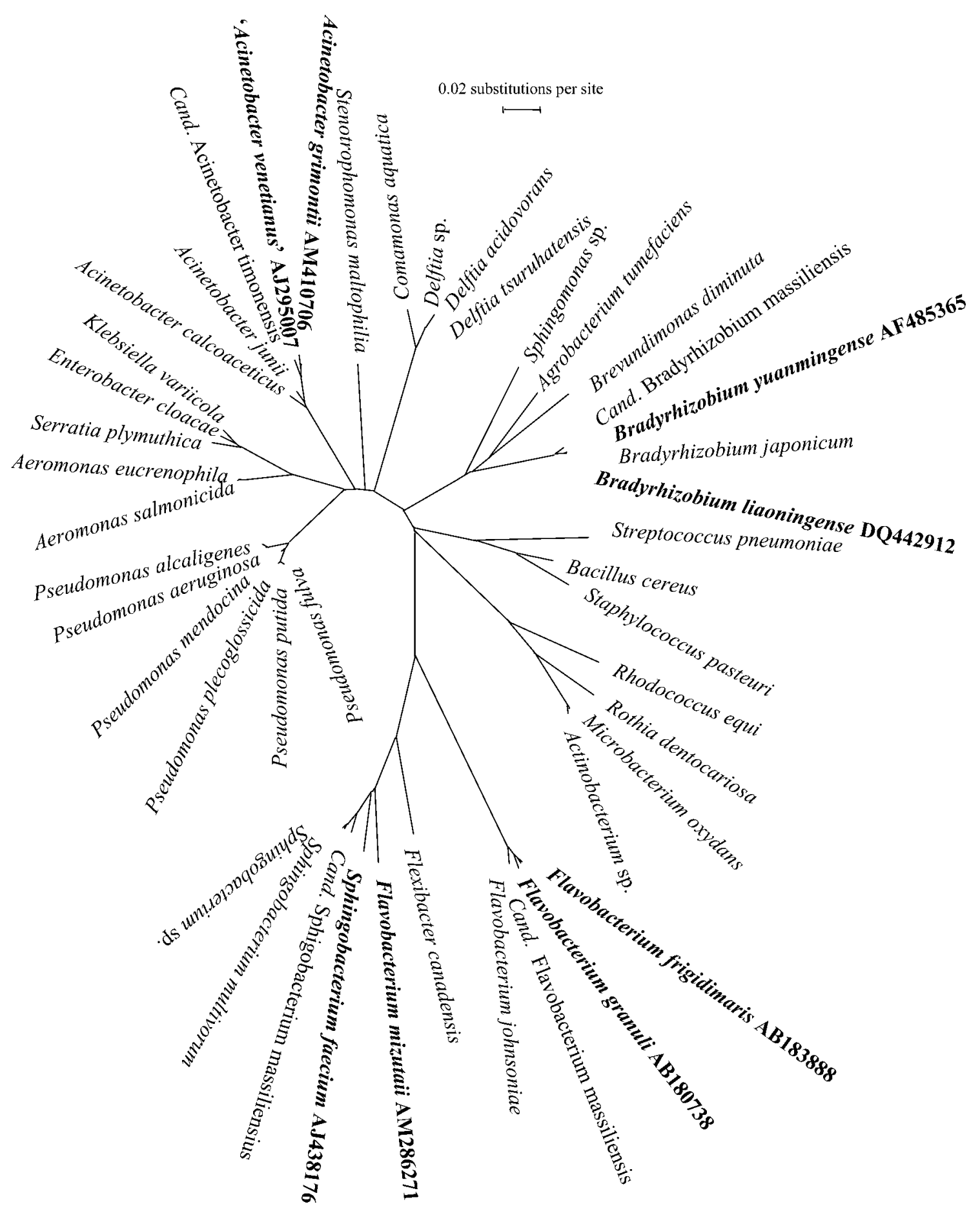

Fig. 1. Phylogenetic tree showing the position of all ARB isolated in this study. The tree was constructed using the neighbourjoining method, based on the partial 16S rRNA gene coding sequence. Taxonomic names or proposed names for Candidatus species are indicated at the ends of the branches. Reference strains with accession numbers are in bold.

communities in soil. Members of these phyla make up $92 \%$ of soil inhabitants, on average (Janssen, 2006). The majority of isolates in this study had $\geqslant 99 \% 16$ S RNA gene similarity with previously characterized species, but four strains exhibited $\leqslant 98 \% 16 \mathrm{~S}$ rRNA gene similarity with known species or genera; these strains belonged to the species Acinetobacter junii, Bradyrhizobium japonicum, Flavobacterium johnsoniae and Sphingobacterium multivorum. These four isolates are likely to be new species or genera (Stackebrandt \& Goebel, 1994), and polyphasic 


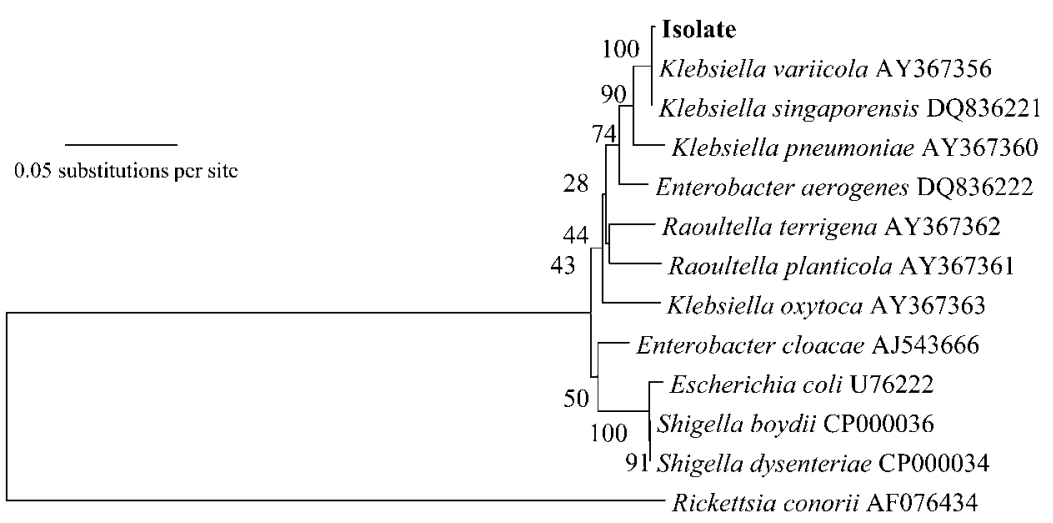

Fig. 2. Phylogenetic tree showing the position of our Klebsiella isolate among other Klebsiella spp. and members of the related genus Raoultella; the bootstraps are marked. The tree was constructed using the neighbourjoining method, based on the partial rpoB gene coding sequence. characterization of other isolates will have to be performed for definitive descriptions of these strains. In contrast to analysis of soil libraries of $16 \mathrm{~S}$ rRNA genes, culture methods open the way for characterization of such isolates.

In our survey, Pseudomonas aeruginosa was able to lyse amoebae more quickly than other isolates (Table 1). The ability of $P$. aeruginosa to resist amoeba predation has been described by others (Wang \& Ahearn, 1997), and it has been demonstrated recently that this lysis is related to necrosis and apoptosis, and mediated by the effect of type III secretion system effector proteins (Abd et al., 2008). The pathogenicity of $P$. aeruginosa in humans has been demonstrated to be associated with resistance to its lytic effect on amoebae (Fenner et al., 2006). Pseudomonas putida also provoked complete amoebal lysis in our study, and this is thought to be the first report of such activity. $P$. putida is rarely pathogenic for humans, but it has been found associated with empyema, urinary tract infections, septicaemia and various other episodes.

Pseudomonas fulva, Microbacterium oxydans, Rhodococcus equi, Actinobacterium sp. and K. variicola (or K. singaporensis) induced complete, but slower, amoebal lysis. Members of the Rhodococcus genus, especially R. equi, are known to be pathogens in immunocompromised patients. $K$. variicola is a recently described species that seems to be genetically isolated from $K$. pneumoniae strains (Rosenblueth et al., 2004). Here, we report what we believe is the first culture of $K$. variicola from soil, and the discovery of its lytic ability towards amoebae. It is known that strains of $K$. variicola are abundant in plants, and that they represent less than $10 \%$ of the clinical Klebsiella isolates that have, in the past, been considered to be $K$. pneumoniae. $K$. variicola seems to be better adapted to the inanimate environment than $K$. pneumoniae, and strains of $K$. variicola from plants can occasionally infect humans (Martinez et al., 2004). Our strain showed equal similarity to K. variicola and to K. singaporensis ( $\mathrm{Li}$ et al., 2004).

Aeromonas eucrenophila, Bacillus cereus, Flexibacter canadensi, Stenotrophomonas maltophilia, and the isolate with $97 \%$ similarity to Bradyrhizobium japonicum, caused partial amoebal lysis. We believe that this is the first report of the lytic ability of these bacteria towards amoebae. However, many Bradyrhizobium spp. have been shown to be ARB in our previous work (Pagnier et al., 2008). Three of the other isolates in the present study could be regarded as potential pathogens. The pathogenicity of $B$. cereus for humans is well-known. Ste. maltophilia (previously known as Pseudomonas maltophilia and Xanthomonas maltophilia) has been associated with various (mostly nosocomial) infections, including bacteraemia, meningitis, wound infections, urinary tract infections and pneumonia. In 2000, there was the first report of the isolation of $A$. eucrenophila from a human source (Albert et al., 2000). Survival of Aeromonas salmonicida has been observed in protozoa of the species Tetrahymena pyriformis (King \& Shotts, 1988), and recently it has been demonstrated that free-living amoeba might play a role as a reservoir of Aeromonas spp. (Rahman et al., 2008).

It is remarkable that we did not isolate Legionella spp. The species of this genus have been mostly isolated from water, but some, especially Legionella longbeachae, have also been obtained from soil samples (Koide et al., 2001; Steele et al., 1990). We do not think that this absence is due to a bias introduced by the technique used, as we have isolated many Legionella spp. from hospital water supplies using our technique. Also, in our soil and sand samples, we did not find ARB, such as Afipia or Bosea spp., that are known to be common in hospital water supplies.

The isolation of Str. pneumoniae from sand samples (Plage de la Pointe Rouge) was a surprising result. In order to verify the result, we repeated the culture using the remainder of the sample that had been frozen, and the same Str. pneumoniae strain was isolated. This confirmed that isolation of the strain was not a result of contamination at the time of inoculation; however, contamination occurring at the time of sampling, or during preparation of sample for inoculation, cannot be excluded. Until recently, Str. pneumoniae was not considered to be an environmental species; however, its isolation from environmental sources has been recently reported (Harakeh et al., 2006). Nevertheless, the results reported by Harakeh et al. (2006) are highly questionable, not only because that was the first study to report that Str. pneumoniae was very 
common in water, but also because all isolates tested were resistant to vancomycin; the latter feature has not been reported for human isolates (Daoud et al., 2006; Haas et al., 2005), except by Harakeh et al. (2006). Our isolate was susceptible to vancomycin. It was isolated in a well containing colistin and vancomycin, and this confirms that the strain grew as an intracellular bacterium within amoebae, as it would have been killed by vancomycin if it had been growing extracellularly.

To summarize, this preliminary work reports that several known human opportunistic pathogens act as ARBs when isolated from soil and sand, and that the soil and sand in the vicinity of humans contain some ARB that are believed to be new species. A limitation of the present work is that we used a single species of amoeba. For example, in the case of members of the genus Legionella, not all species are able to grow in the protozoa that support the growth of the species Legionella pneumophila. It is likely that use of protozoa other than the species Acanthamoeba polyphaga would lead to a slightly different panel of isolates. In future, it may be interesting to study soils of other origins, including samples from regions that are more humid than our city (Marseilles has a dry Mediterranean climate), to investigate the ARB populations of soil in different climatic conditions. Use of different protozoan hosts to perform coculture would also be of interest to give a more accurate evaluation of the panel of ARBs in soil.

\section{REFERENCES}

Abd, H., Weintraub, A. \& Sandstrom, G. (2005). Intracellular survival and replication of Vibrio cholerae $\mathrm{O} 139$ in aquatic free-living amoebae. Environ Microbiol 7, 1003-1008.

Abd, H., Wretlind, B., Saeed, A., Idsund, E., Hultenby, K. \& Sandstrom, G. (2008). Pseudomonas aeruginosa utilises its type III secretion system to kill the free-living amoeba Acanthamoeba castellanii. J Eukaryot Microbiol 55, 235-243.

Adekambi, T., Reynaud-Gaubert, M., Greub, G., Gevaudan, M. J., La Scola, B., Raoult, D. \& Drancourt, M. (2004). Amoebal coculture of 'Mycobacterium massiliense' sp. nov. from the sputum of a patient with hemoptoic pneumonia. J Clin Microbiol 42, 5493-5501.

Adeleke, A. A., Fields, B. S., Benson, R. F., Daneshvar, M. I., Pruckler, J. M., Ratcliff, R. M., Harrison, T. G., Weyant, R. S., Birtles, R. J. \& other authors (2001). Legionella drozanskii sp. nov., Legionella rowbothamii sp. nov. and Legionella fallonii sp. nov.: three unusual new Legionella species. Int J Syst Evol Microbiol 51, 1151-1160.

Albert, M. J., Ansaruzzaman, M., Talukder, K. A., Chopra, A. K., Kuhn, I., Rahman, M., Faruque, A. S., Islam, M. S., Sack, R. B. \& Mollby, R. (2000). Prevalence of enterotoxin genes in Aeromonas spp. isolated from children with diarrhea, healthy controls, and the environment. J Clin Microbiol 38, 3785-3790.

Barker, J. \& Brown, M. R. W. (1994). Trojan horses of the microbial world: protozoa and the survival of bacterial pathogens in the environment. Microbiology 140, 1253-1259.

Birtles, R. J., Rowbotham, T. J., Michel, R., Pitcher, D. G., La Scola, B., Alexiou-Daniel, S. \& Raoult, D. (2000). 'Candidatus Odyssella thessalonicensis' gen. nov., sp. nov., an obligate intracellular parasite of Acanthamoeba species. Int J Syst Bacteriol 50, 63-71.
Brown, M. R. \& Barker, J. (1999). Unexplored reservoirs of pathogenic bacteria: protozoa and biofilms. Trends Microbiol 7, 46-50.

Crawford, R. L. (2005). Microbial diversity and its relationship to planetary protection. Appl Environ Microbiol 71, 4163-4168.

Daoud, Z., Cocozaki, A. \& Hakime, N. (2006). Antimicrobial susceptibility patterns of Haemophilus influenzae and Streptococcus pneumoniae isolates in a Beirut general university hospital between 2000 and 2004. Clin Microbiol Infect 12, 86-90.

Drancourt, M., Roux, V., Fournier, P. E. \& Raoult, D. (2004). rpoB gene sequence-based identification of aerobic Gram-positive cocci of the genera Streptococcus, Enterococcus, Gemella, Abiotrophia, and Granulicatella. J Clin Microbiol 42, 497-504.

Fenner, L., Richet, H., Raoult, D., Papazian, L., Martin, C. \& La Scola, B. (2006). Are clinical isolates of Pseudomonas aeruginosa more virulent than hospital environmental isolates in amebal co-culture test? Crit Care Med 34, 823-828.

Greub, G. \& Raoult, D. (2002). Parachlamydiaceae: potential emerging pathogens. Emerg Infect Dis 8, 625-630.

Greub, G. \& Raoult, D. (2003). Rhodobacter massiliensis sp. nov., a new amoebae-resistant species isolated from the nose of a patient. Res Microbiol 154, 631-635.

Greub, G. \& Raoult, D. (2004). Microorganism resistant to free-living amoebae. Clin Microbiol Rev 17, 413-433.

Greub, G., La Scola, B. \& Raoult, D. (2004). Amoeba-resisting bacteria isolated from human nasal swabs by amoebal coculture. Emerg Infect Dis 10, 470-477.

Haas, W., Kaushal, D., Sublett, J., Obert, C. \& Tuomanen, E. I. (2005). Vancomycin stress response in a sensitive and a tolerant strain of Streptococcus pneumoniae. J Bacteriol 187, 8205-8210.

Harakeh, S., Yassine, H. \& El-Fadel, M. (2006). Antimicrobialresistance of Streptococcus pneumoniae isolated from the Lebanese environment. Mar Environ Res 62, 181-193.

Janssen, P. H. (2006). Identifying the dominant soil bacterial taxa in libraries of 16S rRNA and 16S rRNA genes. Appl Environ Microbiol 72, 1719-1728.

King, C. H. \& Shotts, E. B. (1988). Enhancement of Edwardsiella tarda and Aeromonas salmonicida through ingestion by the ciliated protozoan Tetrahymena pyriformis. FEMS Microbiol Lett 51, 95-99.

Koide, M., Arakaki, N. \& Saito, A. (2001). Distribution of Legionella longbeachae and other legionellae in Japanese potting soils. J Infect Chemother 7, 224-227.

La Scola, B. \& Raoult, D. (2001). Survival of Coxiella burnetii within free-living amoebae Acanthamoeba castellanii. Clin Microbiol Infect 7, 75-79.

La Scola, B., Michel, G. \& Raoult, D. (1997). Use of amplification and sequencing of the $16 \mathrm{~S}$ rRNA gene to diagnose Mycoplasma pneumoniae osteomyelitis in a patient with hypogammaglobulinemia. Clin Infect Dis 24, 1161-1163.

La Scola, B., Barrassi, L. \& Raoult, D. (2000). Isolation of new fastidious $\alpha$ Proteobacteria and Afipia felis from hospital water supplies by direct plating and amoebal co-culture procedures. FEMS Microbiol Ecol 34, 129-137.

La Scola, B., Mezi, L., Weiller, P. J. \& Raoult, D. (2001). Isolation of Legionella anisa using an amoebal coculture procedure. J Clin Microbiol 39, 365-366.

La Scola, B., Boyadjiev, I., Greub, G., Khamis, A., Martin, C. \& Raoult, D. (2003). Amoeba-resisting bacteria and ventilator-associated pneumonia. Emerg Infect Dis 9, 815-821.

La Scola, B., Barrassi, L. \& Raoult, D. (2004a). A novel alphaProteobacterium, Nordella oligomobilis gen. nov., sp. nov., isolated by using amoebal co-cultures. Res Microbiol 155, 47-51. 
La Scola, B., Birtles, R. J., Greub, G., Harrison, T. J., Ratcliff, R. M. \& Raoult, D. (2004b). Legionella drancourtii sp. nov., a strictly intracellular amoebal pathogen. Int $J$ Syst Evol Microbiol 54, 699-703.

Landers, P., Kerr, K. G., Rowbotham, T. J., Tipper, J. L., Keig, P. M., Ingham, E. \& Denton, M. (2000). Survival and growth of Burkholderia cepacia within the free-living amoeba Acanthamoeba polyphaga. Eur $J$ Clin Microbiol Infect Dis 19, 121-123.

Li, X., Zhang, D., Chen, F., Ma, J., Dong, Y. \& Zhang, L. (2004). Klebsiella singaporensis sp. nov., a novel isomaltulose-producing bacterium. Int J Syst Evol Microbiol 54, 2131-2136.

Martinez, J., Martinez, L., Rosenblueth, M., Silva, J. \& MartinezRomero, E. (2004). How are gene sequence analyses modifying bacterial taxonomy? The case of Klebsiella. Int Microbiol 7, 261268.

Mollet, C., Drancourt, M. \& Raoult, D. (1997). $r p o B$ sequence analysis as a novel basis for bacterial identification. Mol Microbiol 26, 10051011.

Molmeret, M., Horn, M., Wagner, M., Santic, M. \& Abu, K. Y. (2005). Amoebae as training grounds for intracellular bacterial pathogens. Appl Environ Microbiol 71, 20-28.

Pagnier, I., Raoult, D. \& La Scola, B. (2008). Isolation and identification of amoeba-resisting bacteria from water in human environment by using an Acanthamoeba polyphaga co-culture procedure. Environ Microbiol 10, 1135-1144.

Rahman, M., Abd, H., Romling, U., Sandstrom, G. \& Mollby, R. (2008). Aeromonas-Acanthamoeba interaction and early shift to a viable but nonculturable state of Aeromonas by Acanthamoeba. J Appl Microbiol 104, 1449-1457.

Rosenblueth, M., Martinez, L., Silva, J. \& Martinez-Romero, E. (2004). Klebsiella variicola, a novel species with clinical and plantassociated isolates. Syst Appl Microbiol 27, 27-35.

Snelling, W. J., Moore, J. E., McKenna, J. P., Lecky, D. M. \& Dooley, J. S. (2006). Bacterial-protozoa interactions; an update on the role these phenomena play towards human illness. Microbes Infect 8, 578-587.

Stackebrandt, E. \& Goebel, B. M. (1994). Taxonomic note: a place for DNA-DNA reassociation and $16 \mathrm{~S}$ rRNA sequence analysis in the present species definition in bacteriology. Int J Syst Bacteriol 44, 846-849.

Steele, T. W., Moore, C. V. \& Sangster, N. (1990). Distribution of Legionella longbeachae serogroup 1 and other legionellae in potting soils in Australia. Appl Environ Microbiol 56, 2984-2988.

Wang, X. \& Ahearn, D. G. (1997). Effect of bacteria on survival and growth of Acanthamoeba castellanii. Curr Microbiol 34, 212-215.

Edited by: G. Muyzer 